\title{
L'effet de la pandémie de la COVID-19 sur Résonances et ses artistes
}

\section{Marjorie Deschamps, Yaëlle Azoulay, Noémie Azoulay}

Au début du mois de mars 2020, alors que certains États commençaient à prendre des mesures plus drastiques pour contrôler l'évolution de la pandémie et que seulement quelques cas étaient déclarés au Canada, nous étions loin de nous douter de l'impact qu'aurait la COVID-19 sur notre industrie, sur notre entreprise, sur la carrière de nos artistes et sur nos vies personnelles. L'annonce de l'interdiction des rassemblements intérieurs de plus de 250 personnes par le gouvernement le 12 mars 2020, puis la série de mesures mises en place par la Santé publique dans les semaines qui suivirent, furent un choc terrible pour notre agence dont le modèle d'affaires reposait essentiellement sur la vente de spectacles de nos artistes. Notre première réaction fut d'essayer de "sauver » le plus d'engagements possible en reportant un maximum de concerts, mais nous nous sommes rapidement aperçues que la crise sanitaire que nous vivions ne s'estomperait pas de sitôt.

Les hauts et les bas ont ainsi débuté. Parfois, des nouvelles encourageantes nous donnaient espoir, et puis c'était l'annulation d'événements d'envergure à l'international qui venait assombrir le tout. Ces montagnes russes émotives ont dû être encaissées de plein fouet par tous nos artistes. Évidemment, tout le monde n'a pas réagi de la même façon. Certains ont été aspirés par le trou noir; d'autres ont eu des élans créatifs; d'autres encore ont pris un temps de réflexion. Notre travail en tant qu'agentes et gérantes repose essentiellement sur le lien que nous entretenons avec nos artistes. Ainsi, dans les circonstances du confinement, nous devions nous assurer de faire un suivi étroit avec chacun d'eux, autant sur le plan émotionnel que professionnel. Nous avons constaté un sentiment assez généralisé d'inquiétude, accompagné de beaucoup d'incertitude et d'un grand niveau d'impuissance. Ces sentiments ont également été partagés par notre équipe, qui a dû se repositionner et apprendre à vivre autrement.

Dans un contexte où il nous était devenu impossible de nous rendre au bureau, nous avons dû rapidement mettre en place des réunions d'équipe virtuelles hebdomadaires. Une des employées a également travaillé sur un plan d'urgence pour nous accompagner dans la gestion des ressources humaines en temps de pandémie et nous sortir de notre paralysie momentanée. De plus, étant cinq femmes dans l'agence, dont quatre avec enfants, nous avons dû composer avec la conciliation travail-famille qui s'est imposée lorsque les garderies et écoles ont dû fermer. Ceci s'est avéré un défi de taille, alors que nous étions en mode survie et devions nous activer en redoublant d'ardeur pour composer avec les reports, annulations, et conséquences dramatiques de la crise sur nos artistes et sur notre plan stratégique de compagnie.

Dès le mois d'avril, un changement de cap s'est effectué dans nos priorités. Au-delà des préoccupations financières et de la survie de la compagnie, nous nous sommes mises en quête d'un projet qui rassemblerait à la fois nos artistes (toutes disciplines confondues) et l'équipe de l'Agence. Cette situation complètement nouvelle a donc généré de l'inspiration pour mettre sur pied un nouveau volet destiné à la captation audiovisuelle de spectacles et à la webdiffusion, domaines dans lesquels nous avions peu de repères, mais qui donnaient de la visibilité à nos artistes et allaient les stimuler sur le plan créatif! Nous nous sommes donc entourées de professionnels et nous sommes lancées (un peu naïvement) dans ce nouveau projet d'envergure : La Trame de Résonances. Durant le printemps, nous nous sommes affairées à trouver les bons partenaires, à mobiliser nos artistes et bien sûr, à envoyer diverses demandes de subvention pour soutenir le projet. Depuis, nous effectuons un à deux tournages par mois, créant ainsi de nouveaux outils pour le développement de nos artistes à court, moyen, et long terme. 
Une préoccupation de longue date ayant été accentuée par la pandémie est sans contredit le fait qu'il y ait peu de diversité dans l'offre de spectacles destinée au grand public. Comme nous travaillons principalement avec des artistes dits " de niche », nous sommes soucieuses par rapport à l'écologie de l'industrie. Nous remarquons une tendance depuis le début de la pandémie au niveau de l'offre de spectacles : les solos ou les duos sont privilégiés et l'on mise sur des têtes d'affiche uniquement. Les artistes œuvrant dans des styles plus nichés en souffrent et l'écologie du milieu artistique n'est pas équilibrée.

Nous pensons que les effets de la pandémie se feront sentir longtemps, tout spécialement sur les artistes qui tournaient principalement à l'international. Avec les contraintes sanitaires actuelles telles que la quarantaine de 14 jours après un séjour à l'étranger, il est difficile de planifier des tournées en dehors du Canada. Nous devons donc revenir à une échelle plus locale à court et moyen termes si nous voulons garder les artistes actifs. Dans un même ordre d'idées, l'équipe de Résonances voyageait fréquemment aux quatre coins du globe afin de représenter ses artistes. Ici aussi, il faut trouver de nouvelles façons de faire pour garder le lien avec les partenaires et les diffuseurs internationaux. Le virtuel ayant parfois ses limites, il est difficile au moment d'écrire ces lignes (août 2020) d'évaluer l'impact à long terme sur notre travail d'agente d'artistes.

Plus récemment, l'équipe de direction de l'Agence s'est réunie afin de se repositionner sur son plan d'avenir et de réfléchir aux fondements de l'agence sous l'influence de la crise que nous traversons. II est ressorti de nos discussions un nouveau système qui guidera nos actions via quatre axes de travail. Nous avons constaté que nous devions restructurer l'organisme pour ne plus être tributaires presque exclusivement de la vente directe de spectacles et des commissions. Nous voulons travailler sur de la diffusion alternative, des projets plus engagés aux niveaux social et communautaire, et des partenariats nouveaux qui ne seraient pas forcément des salles de spectacles. Pour que notre plan fonctionne, nous aurons certainement besoin de nouvelles sources de financement plus diversifiées, de meilleur soutien financier pour nos artistes, de partenariats à long terme et d'une réorganisation de nos ressources humaines. Ce virage s'opérera au fil des prochaines années, en espérant le soutien de nos bailleurs de fonds et de nos artistes.

Par ailleurs, un autre enjeu ayant été accentué par la pandémie est la question de la rétribution des artistes qui génèrent du contenu virtuel. Celui-ci étant devenu leur unique source de revenus, il est impératif de s'assurer que les Facebook, YouTube, Spotify, et autres grands médias de ce monde soient mieux encadrés afin que les lois soutiennent les artistes et atténuent leur précarité. Cette précarité a été exacerbée dans les derniers mois et elle pousse à avoir une réflexion sur le statut de l'artiste et un revenu minimum garanti (possiblement inspiré du modèle de l'intermittence de la France).

En écrivant ces lignes, nous constatons que nous manquons encore cruellement de recul face à la crise puisque nous sommes encore en plein dedans et en vivons les contrecoups au quotidien. Nous ne pouvons que constater le chemin parcouru jusqu'ici, truffé de rebondissements et géré souvent à la dernière minute, en mode panique, et en improvisant quelque peu à chacun des tournants. Certainement, nous développons une résilience qui nous servira dans la poursuite de notre rêve d'affaires ambitieux, celui de donner des ailes aux artistes qui nous sont chers, autant sur la scène locale qu'internationale, en contexte de pandémie ou non! 